\title{
Development and verification of accurate stopping control algorithm with scale models
}

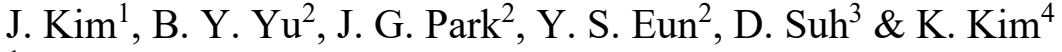 \\ ${ }^{1}$ Korea Railroad Research Institute, Korea \\ ${ }^{2}$ DGIST, Korea \\ ${ }^{3}$ Green System Co., Ltd., Korea \\ ${ }^{4}$ Hanbat National University, Korea
}

\begin{abstract}
Accurate stopping technology that is used to stop a train precisely at a desired position has been continuously studied. Recently, this technology has become more important as platform screen doors have been installed in metro stations for passenger safety. Various algorithms have been proposed for accurate stopping. However, it requires a lot of time and money to verify experimentally the performance of novel algorithms with actual trains. Hence, it is difficult to improve and verify control performance with newly proposed algorithms. To resolve this problem, we propose to build a test bed for the accurate stopping control using scale models of train and track. After analysing the acceleration and deceleration characteristics of a specified train that is going to be equipped with a novel proposed algorithm, an adjuster is added to make the acceleration and deceleration characteristics of the scale model be similar to those of the target train. System identification was also performed by analysing the step response with various step input to the scale model. In this study, feedforward with proportional and integral (PI) controller was proposed, where gain parameters were determined based on the system characteristics analysed by experiments. The structure of the test bed and the process of building the test bed for the accurate stopping control are described in this paper. Experiment results are also provided to show the performance of the proposed controller. By being tested with this test bed, an improved control algorithm for the accurate stopping is expected to be derived.
\end{abstract}

Keywords: accurate stopping, scale model, test bed, feedforward control. 


\section{Introduction}

The efficiency of train scheduling is a major issue of the urban rail transit because it is highly related to the citizens' convenience. Therefore, many studies and efforts have been conducted to increase the efficiency. Enlarging the door width of railway vehicle is one of methods to increase the efficiency because it decreases the boarding and alighting time of passengers [1]. To enlarge the door width of railway vehicle, it is considered the structural stability of the rolling stock as well as the stopping accuracy especially if platform screen door (PSD) is installed in the station. The train should stop in a range of accuracy that the vehicle door and PSD should be aligned, and the accuracy is determined by the difference between the widths of door and PSD. For example, the width of PSD installed in the heavy rail station in Korea is $2,000 \mathrm{~mm}$. And that of door in the railway vehicle is $1,300 \mathrm{~mm}$. Hence, the train must stop to the platform within the error of $\pm 350 \mathrm{~mm}$. If the door is going to be enlarged, the stop control needs strict accuracy.

Accordingly, many methods have been studied and proposed to increase stopping accuracy. Because the traction and braking are essential part in accurate stop control, many researchers focussed on the traction and braking in a train. Ahmad [2] applied model reference adaptive control to estimate braking distance by braking control. Song et al. [3] applied backstepping control adaptively to traction and braking in the high speed railway. There is also a study on modelling and system identification of the braking system of urban rail vehicles [4]. Modelling is the first step to build a controller, and some studies focussed on modelling of a railway vehicle. Chen and Gao [5] proposed a model to estimate to stop error, where subtractive clustering method was chosen to generate an adaptive network based fuzzy inference system. Chen et al. [6] also proposed to use machine learning with balises for the accurate stop. Dong et al. [7] developed a numerical model of high-speed trains in China and simulated the automatic train control system.

The train is a complicated and composite system; hence it is difficult to analyse how the proposed method works in the system. Besides, it is also difficult to verify and to improve the method by performing various trials. In this studies, we propose to build a test bed using scale models of train and track. Actually these models are usually used as a toy or as used to display the operation of transit system. However, we use scale models in the control area. To do this, mechanical and dynamical properties of both a real train and a scale model should be considered and the similarity between them should be guaranteed to reasonable level. This is called as similitude. Then the system should be identified and proper control algorithm should be designed. We should find proper gain parameters in the controller with many trials.

This paper describes the process of designing the controller as well as building the test bed and is organized as follows: First, the organization of the test bed is described in section 2 . We show the physical properties of scale model and the measurement and control process in the test bed. Next, the process of system identification is described in section 3. Then, the controller design is described in 
section 4. Test result is described in section 5. Finally, we conclude and remark about further research in section 6 .

\section{Organization of test bed}

In this section, we show how the test bed for the accurate stopping control is organized. The physical properties as well as the structural properties are described. The mechanical and electrical structures in measuring and controlling the speed of train model is also described.

\subsection{Structure of test bed}

The test bed is composed of mechanical part and electrical part. In the mechanical part, there are scale models of train and track. We used an O-scale model. There are many types of models and these are usually categorized by the ratio of a model to the real train or by the size of gauge which is the distance between two loadbearing rails [8]. There is another rail (the third rail) which is used to transmit speed commands. The ratio of O-scale model is 1:48 in USA [8]. The track was also built in line with the same scale, where the train model runs on. Figure 1 shows the overall test bed. We can see whole parts in the test bed.

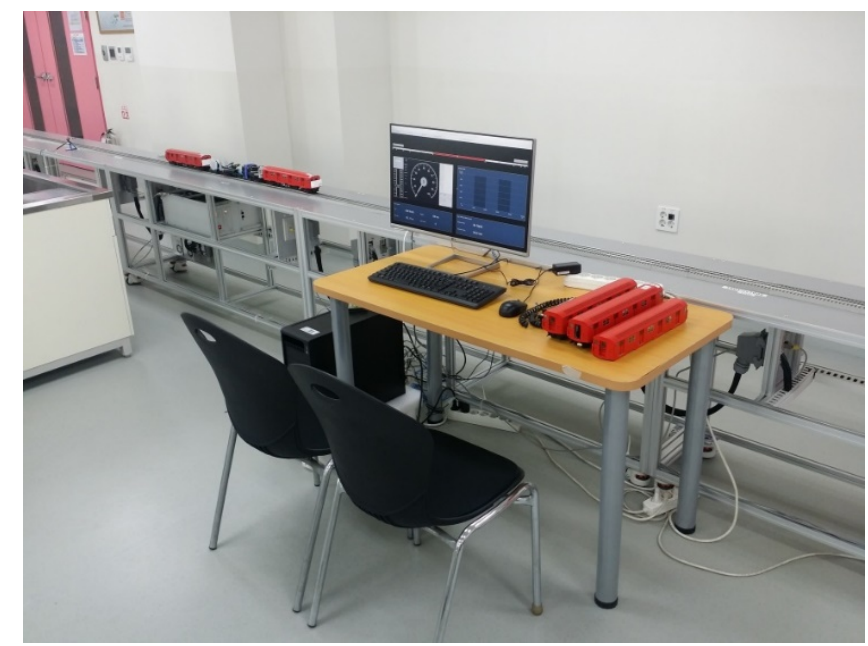

Figure 1: The test bed with scale models of train and track.

Control system, speed detect system, and range detect system are included in the electrical part. The control system can be divided into an operation control system and a model controller. The model controller controls the train scale model by adjusting the voltage level to the motor. And the operation control system simulates ATP (Automatic Train Protector) and ATO (Automatic Train Operator) in the real system which generates the speed profile and decides to accelerate or to decelerate the train. Speed detect system is designed to measure the real speed 
using an external encoder attached on an additional trailer, and range detect system is designed to measure the stop error using a laser sensor. The functional structure of test bed is represented in Figure 2, and each component will be described in the next subsection.

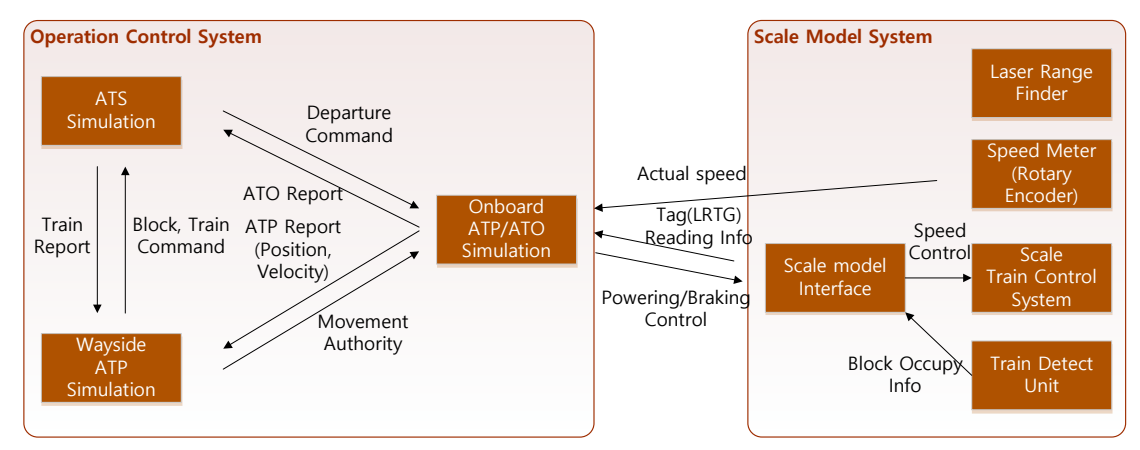

Figure 2: The functional structure of the test bed.

\subsection{Operation control system}

The operation control system simulates ATP and ATO in the real railway system. These are embodied in a workstation. The function of ATP and ATO are separately realized as a software program runs in the workstation. ATP determines the moving authority and the speed limitation of a train while ATO runs the train with regard to the speed profile generated by the ATP. The ATO determines the acceleration level or the deceleration level to run the train. A console monitor is provided where the current state of the scale model is displayed.

Essential information for the train control is exchanged between the operation control system and the scale model system. A command for the acceleration or deceleration level is transmitted to the model controller through the scale model interface. This communication is performed by serial interface. Besides these control communication, two inputs are transmitted to the operation control system. One is speed measured by the external rotary encoder. This input is used to estimate the state variable of train system for better control. The other is the stop error measured by the laser range finder. This information is not used for control but used for display in console to the operator. These two inputs are transmitted through wireless LAN.

\subsection{Scale model system}

The scale model system consists of the scale models of train and track, the model controller, and additional devices attached to the train and track. These components are described in following subsections. 


\subsubsection{Scale models}

Scale models are train and track in the reduced scale (O-scale) which are described in the previous subsection. Motor controller is embedded in the scale model, and it controls the motor by adjusting the DC voltage level. The control command is given by the model controller through the third rail. Track model built in line has a length of $13.6 \mathrm{~m}$ which is equivalent to $652.8 \mathrm{~m}$ in real dimension. The first $2 \mathrm{~m}$ is used for the acceleration of train model and next region is used for the deceleration and stop. The shape of scale models of train and track in the test bed are shown in Figure 3 and Figure 4, respectively.

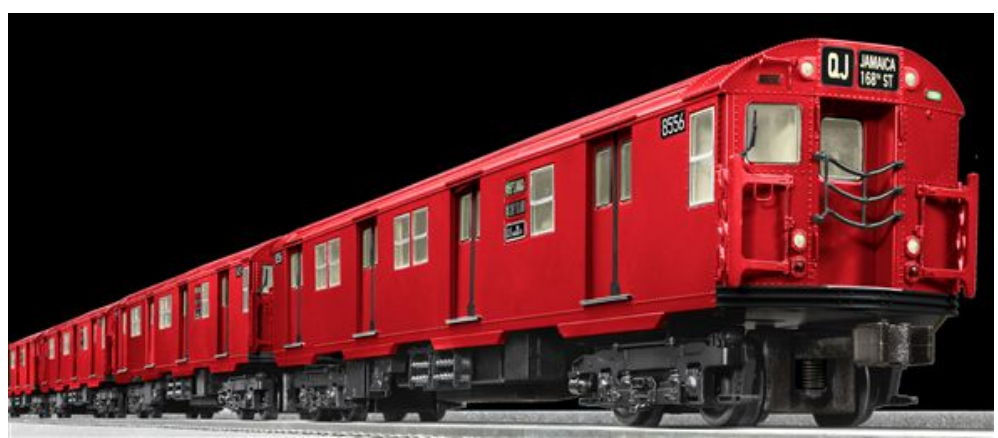

Figure 3: Scale model of a train.

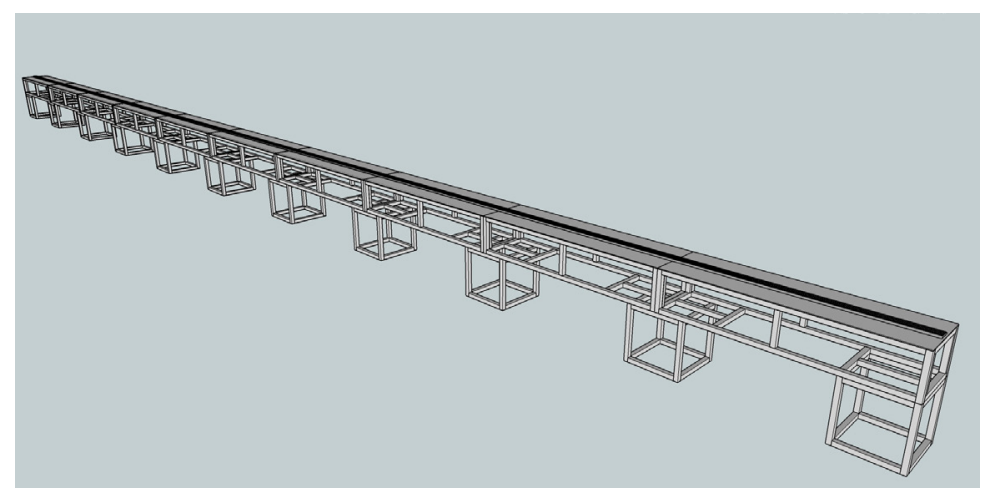

Figure 4: Scale model of track.

\subsubsection{Model controller}

Model controller is made by the model maker, and it controls the motors in the scale model by sending the speed code in PWM. It detects the location of the train by sensing the voltage level between two rails, which is similar method to that of a rail circuit. Accordingly, the controller knows only block occupancy information rather than detail location and this information is transmitted to the operation control system. 


\subsubsection{Speed detect system}

Speed detect system measures the speed of train by an external encoder which is attached on an additional trailer (see Figure 5). Though an internal encoder is installed in the vehicle body of the scale model, the count from the internal encoder may differ from the distance that the train exactly run due to slip between train and track. Hence an external encoder is provided. The encoder pulse is counted by the Arduino board installed on the scale model and counted number is transmitted to the operation control system. The number is used to calculate the speed.

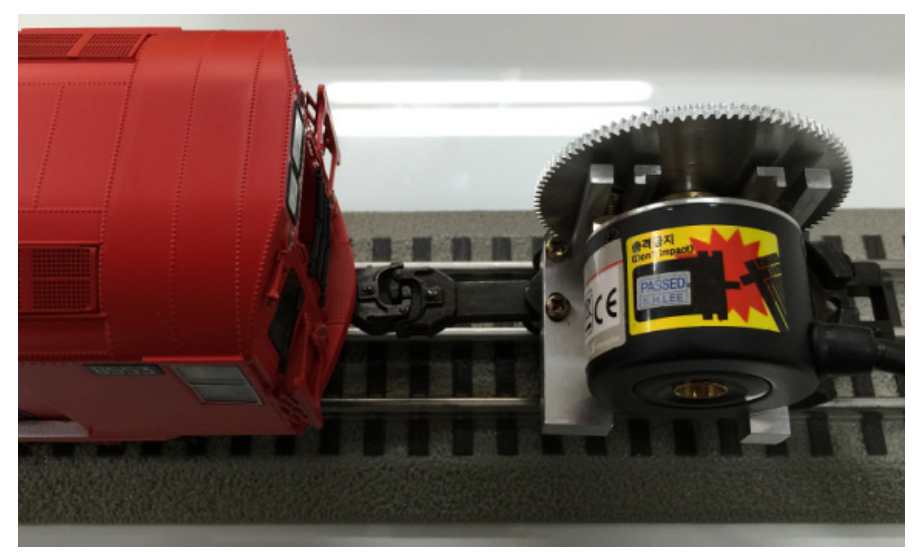

Figure 5: External encoder on an additional trailer.

\subsubsection{Range detect system}

Range detect system is composed of a laser sensor and a wireless transmitter. Laser sensor measures the distance from the sensor to the front end of the train. Therefore, the stop error can be calculated by comparison the location where the train actually stopped with the location where the train should stop. The stop error is displayed on the console of operation control system. The components in the range detect system is displayed in Figure 6.

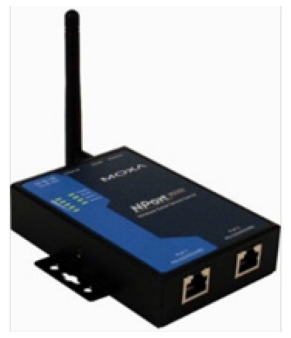

wireless transmitter

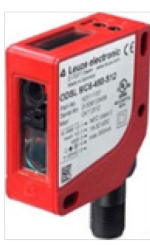

laser range finder

Figure 6: Range detect system consist of wireless transmitter and laser range finder. 


\section{System identification}

The system characteristics of the train scale model was identified by analysing the step input response. We put step input of various level from 5 to 65 to the scale model. It is given to the scale model with PWM code. In other words, the PWM code is changed from zero level to the level that is designated to be investigated. This level change is used to identify the system when it is accelerating. Then the PWM code of zero level is put and the system of decelerating is identified. The step response with various input level is represented with wide grey line in Figure 7.

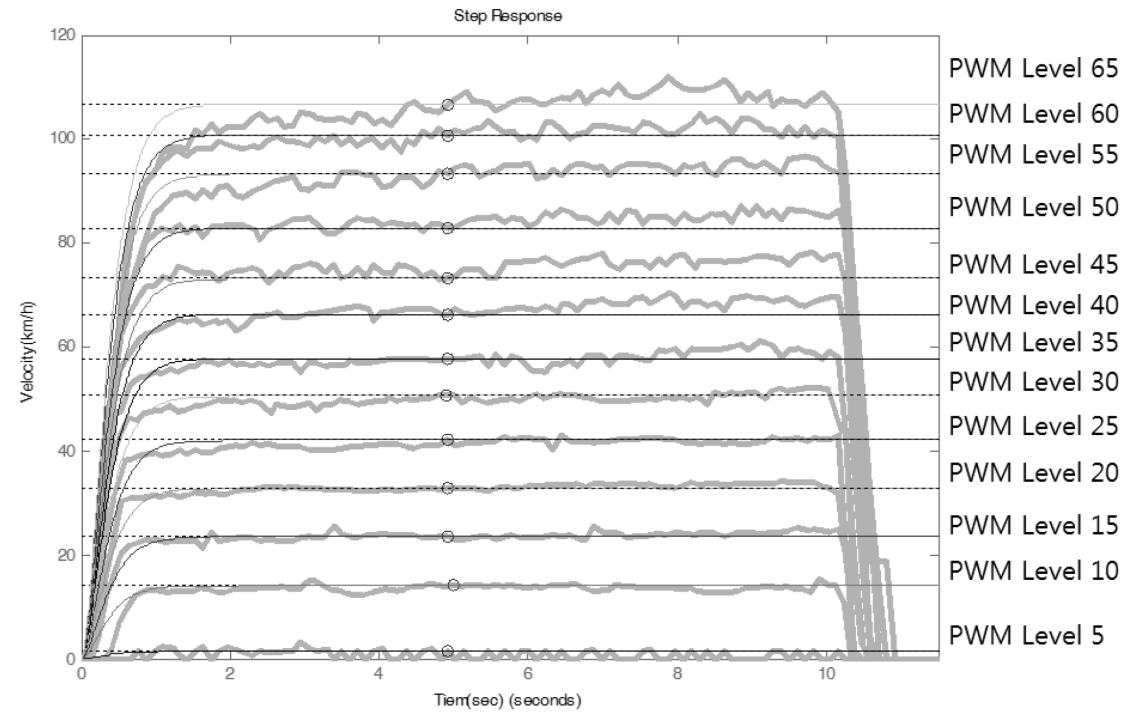

Figure 7: Step responses from various step inputs.

From the step response, we can approximate the system to be a simple mathematical model. Overshoot, rising time, falling time, and etc. are used to decide a proper mathematical model. In this case, the train scale model can be assumed to be a standard 2nd order system that has a double root at -6.5 . The transfer function of the train scale model can be represented as follows:

$$
G_{t}(s)=\frac{6.5^{2}}{(s+6.5)^{2}} .
$$

The step responses with this transfer function (equation (1)) for the various PWM code input with specified level are displayed with narrow solid line in Figure 7. 


\section{Controller design}

Even though the system equation identified in the previous section is stable system, a controller should be designed and attached to the system for the better accuracy.

The controller is composed of speed profile generator, adjuster, quantization, and feed forward plus PI controller. These are organized as depicted in Figure 8. Each component will be explained in next subsection.

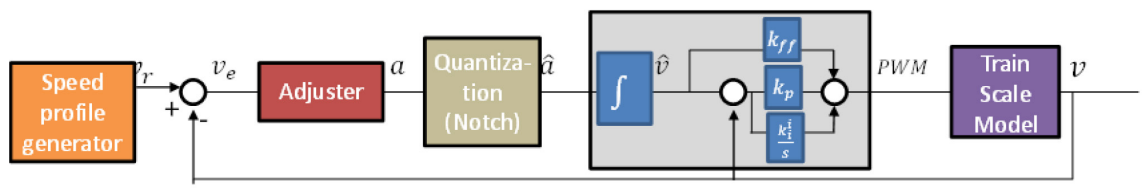

Figure 8: The controller structure.

\subsection{Speed profile generator}

In the stop accuracy control problem, a train runs according to a speed curve that is defined as a function of location. The speed profile is generated by calculating from the desired decelerating rate and the distance to go. Figure 9 shows a typical speed profile. In that figure, there are 4 position stop markers (PSM) at the designated location. Controller uses PSMs to detect correct location of train and new speed profile (Speed Profile 2 in Figure 9) is generated when the train passes a PSM and it finds that localization error is exists. In the scale model, PSM is realized by an infrared sensor.

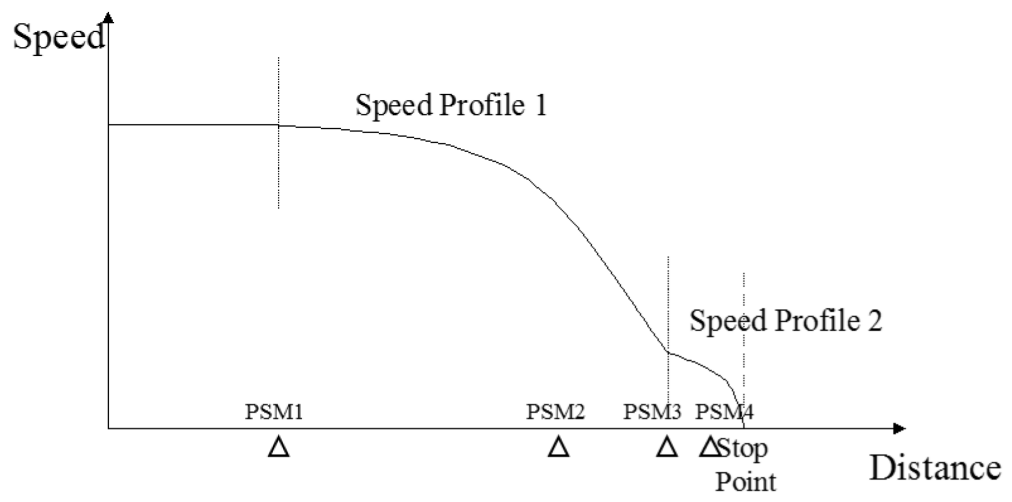

Figure 9: A typical speed profile and PSMs.

\subsection{Adjuster}

The adjuster calculates the desired acceleration/deceleration rate from the reference speed $v_{r}$ and the actual speed $v$. The calculated acceleration/deceleration rate may be out of range that the real train can generate. Furthermore, we should 
convert the rate in the scale model dimension. The ratio of physical size is 1:48, but the ratio of the weight or that of the power is not the same. We should consider all these factors and find the correct acceleration/deceleration rate in the model scale. This is called as similitude and the calculation to match similitude can be performed by dimensionless transformation [9]. In this case, we can obtain the relation between physical units (length, mass, power, and time) by Newton's second law $F=m a$. And if $\lambda_{m}, \lambda_{x}, \lambda_{F}$, and $\lambda_{t}$ denotes the scaling factor of mass, length, power, and time respectively, the relation between scaling factors is represented as follows:

$$
\lambda_{m} \lambda_{x}=\lambda_{F} \lambda_{t}^{2}
$$

The scaling factor of time in the test bed is $1: 1$. Hence, the scaling factor of power between scale model and the real train should be adjusted to be the product of scale factors of mass and length, and the adjustment is performed in the adjuster in Figure 8. Because the mass is fixed, the adjustment of power is fulfilled by adjusting the acceleration/deceleration.

\subsection{Quantization}

The acceleration and deceleration commands are given by setting the notches in the control panel. There are 28 notches of deceleration in the target train. Therefore the adjusted acceleration is quantized to be one of notches. And this becomes the target acceleration/deceleration.

\subsection{Feedforward plus PI controller}

The feedforward plus PI controller is a control block that controls the speed of the scale model. The feedforward control is usually used when the presence of disturbance and the properties of it can be estimated [10]. In this system, braking system has delay and response time, and the feedforward controller compensates these timing disturbances. The PI (proportional and integral) controller is a feedback controller that follows desired output and reduces steady state error.

It is important to assign proper gain for each control block $\left(K_{f f}, K_{p}, K_{i}\right)$ because the control performance depends on these gains. Proper values for these gains were obtained from the system characteristics analysis as well as trial and error, and these are as follows:

$$
K_{f f}=1, K_{p}=1, K_{i}=0.5 \text {. }
$$

Then, the transfer function of the PI controller can be expressed as

$$
C=\frac{s+0.5}{s},
$$

and the transfer function of whole system can be expressed as 


$$
G_{s}=\frac{G_{t}\left(K_{f f}+C\right)}{1+G_{t} C}=\frac{676 s+169}{8 s^{3}+104 s^{2}+676 s+169} .
$$

There are three poles on $-6.3699 \pm 6.3726 i,-0.2602$ and one zero on -0.25 . Because all poles are on the left half plane, these system is stable.

\section{Experiments}

In the experiments section, we conducted 10 trials with the test bed both by the proposed controller and by the $\mathrm{P}$ (proportional) controller only. In that case, the gain of $\mathrm{P}$ controller was set to be one. Besides, the adjuster and the quantization block were not equipped to the $\mathrm{P}$ controller.

The results are represented in Table 1 and Figure 10. The train with P controller tends to overrun because it does not compensate the brake delay. Besides the overrun error, the proposed reduced range and standard deviation of stop position error. Figure 10 shows stop position error with the proposed controller. Two base line pairs at $\pm 7.3 \mathrm{~mm}$ and $\pm 4.2 \mathrm{~mm}$ are drawn addition to the error. These represent $\pm 35 \mathrm{~cm}$ and $\pm 20 \mathrm{~cm}$ in 1:48 scale, respectively. The proposed controller meets the $\pm 35 \mathrm{~cm}$ limit in all trials. For $\pm 20 \mathrm{~cm}$ limit, the proposed controller meets in 8 trials.

This shows that proposed controller represents better performance and thus this may be used in the small tolerance with more improvement.

Table 1: Experimental results (stop position error) of 10 trials with $\mathrm{P}$ controller and proposed controller (unit: $\mathrm{mm}$ ).

\begin{tabular}{|c|c|c|}
\hline Trial/Controller & P controller & Proposed controller \\
\hline 1st & 76.3 & 0.6 \\
\hline 2nd & 80.7 & 0.6 \\
\hline 3rd & 70.8 & 3.9 \\
\hline 4th & 61.7 & -2.1 \\
\hline 5th & 66.4 & 2.8 \\
\hline 6th & 70.8 & -3.6 \\
\hline 7th & 62.9 & 1.5 \\
\hline 8th & 61.1 & 5.2 \\
\hline 9th & 58.3 & 5.3 \\
\hline 10th & 66 & -0.5 \\
\hline Avg. & 67.5 & 1.37 \\
\hline Std. Dev. & 6.7625 & 2.8284 \\
\hline Max & 80.7 & 5.3 \\
\hline Min & 58.3 & -3.6 \\
\hline Range(Max-Min) & 22.4 & 8.9 \\
\hline
\end{tabular}




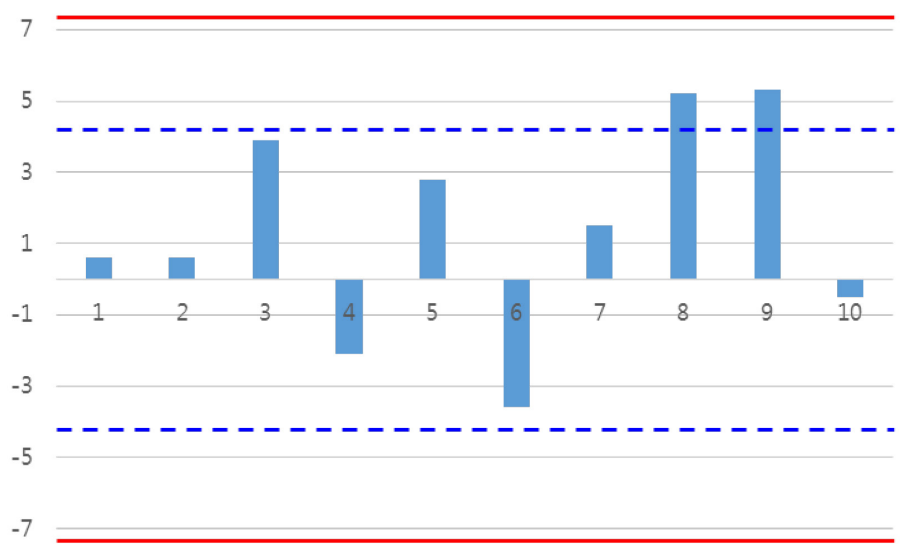

Figure 10: Graphical expression of experimental results with proposed controller and two base line pairs. (red solid line: $\pm 7.3 \mathrm{~mm}$, blue dotted line: $\pm 4.2 \mathrm{~mm})$.

\section{Conclusions and further works}

We proposed development and verification method for accurate stopping control algorithm using scale models. To make the model similar to real railway system, both structural and physical factors were considered. Major software functions were realized in the control system, and similitude was guaranteed by the adjuster attached additionally in the control block. Proposed feedforward plus PI controller represents acceptable accuracy. To develop more accurate controller, mathematically detail modelling and controller design are planned. By performing this work, an improved control algorithm for the accurate stopping is expected to be derived. Furthermore, the validity of verification using scale model is going to be proved.

\section{Acknowledgement}

This research was supported by a grant from R\&D Program of the Korea Railroad Research Institute, Republic of Korea.

\section{References}

[1] Daamen, W., Lee, Y.-C. \& Wiggenraad, P. Boarding and alighting experiments: overview of setup and performance and some preliminary results. Transportation Research Record, (2042), Transportation Research Board of the National Academies, Washington, DC, pp. 71-81, 2008.

[2] Ahmad, H.A. Dynamic braking control for accurate train braking distance estimation under different operating conditions, PhD Thesis, Virginia Polytechnic Institute and State University, 2013. 
[3] Song, Q., Song, Y. \& Cai, W. Adaptive backstepping control of train systems with traction/braking dynamics and uncertain resistive forces, Vehicle System Dynamics, 49(9), pp. 1441-1454, 2011.

[4] Yu, Z. \& Chen, D. Modeling and system identification of the braking system of urban rail vehicles, Journal of the China Railway Society, 33, pp. 37-40, 2011.

[5] Chen, D. \& Gao, C. Soft computing methods applied to train station parking in urban rail transit, Applied Soft Computing, 12, pp. 759-767, 2012.

[6] Chen, D., Chen, R., Li, Y. \& Tang, T. Online learning algorithms for train automatic stop control using precise location data of balises, IEEE Transactions on Intelligent Transportation System, 14(3), pp. 1526-1535, 2013.

[7] Dong, H., Ning, B., Cai, B. \& Hou, Z. Automatic train control system development and simulation for high-speed railways, IEEE Circuits and Systems Magazine, 10, pp. 6-18, 2010.

[8] Wikipedia, https://en.wikipedia.org/wiki/O_scale\#Scale.

[9] Sonin, A.A. The physical basis of dimensional analysis, Department of Mechanical Engineering, MIT, Cambridge, MA, 2001.

[10] Bequette, B.W. Process Control: Modeling, Design, and Simulation, Upper Saddle River, New Jersey, pp. 313-343, 2003. 